\title{
Erratum to: A flow cytometry-based reporter assay identifies macrolide antibiotics as nonsense mutation read-through agents
}

\author{
Michal Caspi $^{1}$ - Anastasia Firsow ${ }^{1}$ - Raja Rajkumar ${ }^{1}$ Nir Skalka ${ }^{1}$ Itay Moshkovits ${ }^{1}$. \\ Ariel Munitz ${ }^{1}$ - Metsada Pasmanik-Chor ${ }^{2} \cdot$ Hagar Greif $^{3}$ - Dalia Megiddo ${ }^{3}$. \\ Revital Kariv $^{4}$ - Daniel W. Rosenberg ${ }^{5} \cdot$ Rina Rosin-Arbesfeld $^{1}$
}

Published online: 20 February 2016

(C) Springer-Verlag Berlin Heidelberg 2016

Erratum to: J MolMed

DOI: 10.1007/s00109-015-1364-1

The original publication of this paper contains a mistake.

The correct name of the 2 Authors should be Itay Moshkovits and Dalia Megiddo.

The online version of the original article can be found at http://dx.doi.org/ 10.1007/s00109-015-1364-1.

Rina Rosin-Arbesfeld

arina@post.tau.ac.il

1 Department of Clinical Microbiology and Immunology, Sackler School of Medicine, Tel Aviv University, Ramat-Aviv, 69978 Tel Aviv, Israel

2 Bioinformatics Unit, G.S.W. Faculty of Life Science, Tel Aviv University, Tel Aviv, Israel

3 Bioblast Pharma, Tel Aviv, Israel

4 Department of Gastroenterology, Tel Aviv Sourasky Medical Center, Tel Aviv 64239, Israel

5 Center for Molecular Medicine, University of Connecticut School of Medicine, Farmington, CT, USA 